\title{
Lunar Surface Propagation Modeling and Effects on Communications
}

\author{
Shian U. Hwu ${ }^{1}$, Matthew Upanavage ${ }^{2}$, and Catherine C. Sham ${ }^{3}$ \\ ${ }^{1}$ Barrios Technology, Houston, TX 77058 \\ ${ }^{2}$ ERC Inc., Houston, TX 77058 \\ ${ }^{3}$ NASA Johnson Space Center, Houston, TX 77058
}

\begin{abstract}
This paper analyzes the lunar terrain effects on the signal propagation of the planned NASA lunar wireless communication and sensor systems. It is observed that the propagation characteristics are significantly affected by the presence of the lunar terrain. The obtained results indicate that the terrain geometry, antenna location, and lunar surface material are important factors determining the propagation characteristics of the lunar wireless communication systems. The path loss can be much more severe than the free space propagation and is greatly affected by the antenna height, operating frequency, and surface material. The analysis results from this paper are important for the lunar communication link margin analysis in determining the limits on the reliable communication range and radio frequency coverage performance at planned lunar base worksites.
\end{abstract}

Key Words-lunar, multipath, path loss, propagation, wireless.

\section{INTRODUCTION}

In recent years, many radio wave propagation studies were conducted using both experimental and theoretical techniques. However, most of these studies were in support of commercial cellular phone wireless applications. The signal frequencies are mostly at the commercial cellular and personal communications services (PCS) bands of 900 and $1800 \mathrm{MHz}$. The antenna configurations are, primarily, one on a high tower and one near the ground to simulate communications between a cellular base station and a mobile unit.

There is great interest in wireless communication and sensor systems for lunar missions by NASA because of the emerging importance of establishing permanent lunar human exploration bases. Since the specific lunar terrain geometries and radio frequencies (RFs) are of interest to the NASA missions, much of the published literature for the commercial cellular and PCS bands of 900 and $1800 \mathrm{MHz}$ may not directly apply to the lunar base environment. Test data from Earth terrain may not be applicable due to foliage/vegetation effects. There are various communication and sensor configurations in a lunar base, including the communications between astronauts, between astronauts and the lunar vehicles, and between lunar vehicles and satellites on the lunar orbits. Also, wireless sensor systems exist among various scientific, experimental sensors, and data collection ground stations.

Lunar communication systems operate in the ultra-high frequency (UHF), S-, X-, and Ka-bands. They are different from the commercial cellular and PCS bands. This study performs multipath and propagation analysis in the lunar environment for the wireless communication and sensor systems at NASA frequency bands. Due to the reflections and diffractions off the surrounding terrain environment, RF signals arrive at a receiver from different directions with different strengths, time delays, and polarization. Thus, a receiver at one location may have a signal strength that is quite different from a similar receiver located only a short distance away. When an astronaut or a lunar vehicle moves from one location to another, significant signal fluctuations may occur depending on the surrounding environment.

Various propagation models were developed to characterize the terrain and environment effects. There are many empirical propagation models for predicting propagation characteristics in Earth rural and urban environments [1]-[6]. Most of the models are based on fitting regression curves to limited measured data, which were collected for specific ranges of various system parameters. These models are not valid for systems with different parameters, such as terrain type, frequencies, and antenna height and configurations, from which the data were collected. No one propagation model can account for all parameter variations for a practical system. The limitations of the models must be considered in applications to achieve a valid design of a wireless system.

The reflections and diffractions are dominating mechanisms in the specific terrain effects at UHF and above frequencies. The Geometrical Theory of Diffraction (GTD) [7]-[12] is capable of taking into account the reflections and diffractions off three-dimensional (3-D) lunar terrain. For detail and specific terrains and objects around the antenna, such as astronauts and the lunar vehicle, we propose using GTD to provide more accurate and reliable results. This paper presents the propagation characteristic analysis of the NASA lunar wireless communication and sensor systems, taking into account the 3-D terrain multipath effects. 


\section{COMPUTATIONAL METHOD}

To determine the signal strength and distribution, including the lunar terrain effects, computer simulations were performed using the computational electromagnetic technique - GTD [7]-[12]. The RF coverage may be determined from computed results and receiver thresholds. At high frequencies, the scattering fields depend on the electrical and geometrical properties of the scatterer in the immediate neighborhood of the point of reflection and diffraction. In the field computation, the incident, reflected, and diffracted fields are determined by the field incident on the reflection or diffraction point multiplied by a dyadic reflection or diffraction coefficient, a spreading factor, and a phase term. The reflected and diffracted fields at a field point r', Er,d(r'), in general, have the following form:

$$
\boldsymbol{E}^{\mathrm{r}, \mathrm{d}}\left(\mathrm{r}^{\prime}\right)=\boldsymbol{E}^{\mathrm{i}}(\mathrm{r}) \boldsymbol{D}^{\mathrm{r}, \mathrm{d}} \boldsymbol{A}^{\mathrm{r}, \mathrm{d}}(\mathrm{s}) \mathrm{e}^{-\mathrm{jks}} .
$$

where $\mathrm{E}^{\mathrm{i}}(\mathrm{r})$ is the field incident on the reflection or diffraction point $r, \boldsymbol{D}^{\mathrm{r}, \mathrm{d}}$ is a dyadic reflection $\left(\boldsymbol{D}^{\mathbf{r}}\right)$ or diffraction $\left(\boldsymbol{D}^{\mathrm{d}}\right)$ coefficient, $\boldsymbol{A}^{\mathrm{r}, \mathrm{d}}(\mathrm{s})$ is a spreading factor for reflection or diffraction, and $s$ is the distance from the reflection or diffraction point $r$ to the field point $r$ '. $D^{\mathrm{r}, \mathrm{d}}$ and $A^{\mathrm{r}, \mathrm{d}}$ can be found from the geometry of the structure at reflection or diffraction point $r$ and the properties of the incident wave there. Thus, the total fields $\left(E^{\text {tot }}\right)$ can be obtained by summing up the individual contributions of the direct field $\left(\mathrm{E}^{\mathrm{dir}}\right)$, reflected field $\left(\mathrm{E}^{\mathrm{ref}}\right)$, and diffracted field $\left(\mathrm{E}^{\mathrm{dif}}\right)$ along the propagation paths, as shown in Fig. 1,

$$
E^{t o t}=E^{d i r}+\sum_{n=1}^{N} E_{n}^{r e f}+\sum_{m=1}^{M} E_{m}^{d i f}
$$

$\mathrm{E}^{\text {tot }}$ : total field at the observation point,

$\mathrm{E}^{\mathrm{dir}}$ : direct fields from antennas,

$\mathrm{E}^{\text {ref }}$ : reflected fields from plates and curve surfaces,

$E^{\text {dif }}$ : diffracted fields from plates and curve surfaces,

where $n$ is the $n^{\text {th }}$ reflection and $\mathrm{N}$ is the total number of reflections. The $\mathrm{m}$ is the $\mathrm{m}^{\text {th }}$ diffraction and $\mathrm{M}$ is the total number of diffractions.

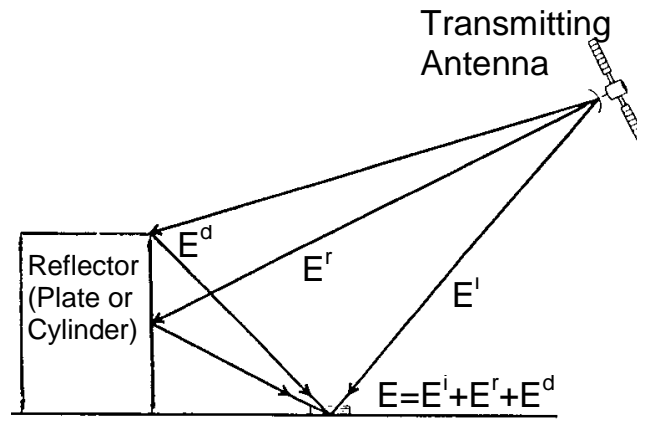

Fig. 1. The GTD field computation.

\section{Flat GROUND SURFACE}

In this section, a simple flat lunar ground surface is investigated. Various RF parameters, such as ground material, operating frequencies, and antenna heights, are investigated for the effects on wireless signal propagation. The signal variations due to various antenna height and lunar surface conductivity were investigated. A lossless (or low-loss) and a lossy (or high-loss) lunar surface material were compared with free space and perfect electric conducting (PEC) ground. For the low loss lunar material, the following parameters are assumed: Relative Permittivity=3, Conductivity=1.E- $11 \mathrm{~S} / \mathrm{m}$. For the high-loss lunar material, the Permittivity $=3$ and Conductivity=1.E-1 $\mathrm{S} / \mathrm{m}$. Both the transmitter and receiver antennas are assumed to be the typical vertical half-wave dipole. The transmitting power is normalized to 1 Watt. Two signals of $\mathrm{UHF}$ at $401 \mathrm{MHz}$ and S-band at $2.1 \mathrm{GHz}$ are investigated. Three antenna heights are analyzed. The $2 \mathrm{~m}$ above ground is used for astronaut personnel communications. The $6 \mathrm{~m}$ and $10 \mathrm{~m}$ are assumed for the lunar vehicle wireless communication systems.

\section{A. Antenna Pattern}

Fig. 2 shows the lunar ground effects on a dipole antenna pattern at $401 \mathrm{MHz}$. The lunar ground is assumed with Permittivity $=3$ and Conductivity=1.E- $1 \mathrm{~S} / \mathrm{m}$. The ground reflections cause the antenna pattern's many ripples with peaks and valleys. Figs. 3 and 4 show the 3-D patterns with and without the lunar ground effects. The ground effects on the antenna pattern are very significant. The lunar ground acts as a partial reflector and partial absorber to the RF signals.

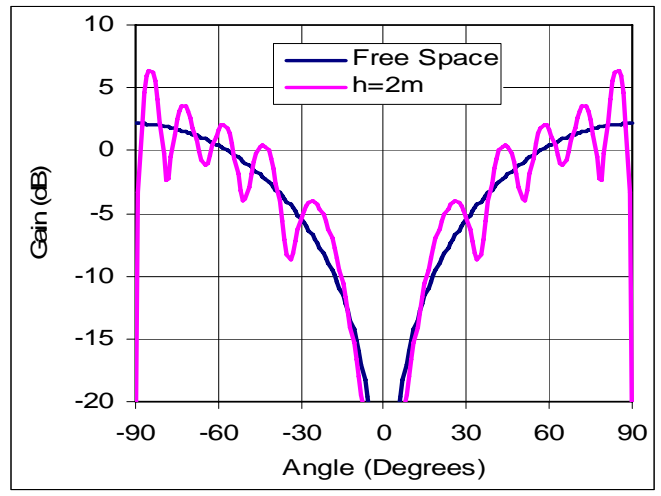

Fig. 2. The lunar ground effects on a dipole antenna pattern at $401 \mathrm{MHz}$. 


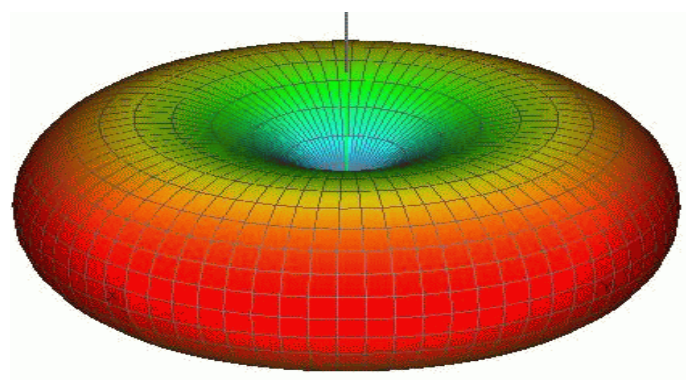

Fig. 3. The free space dipole antenna pattern.

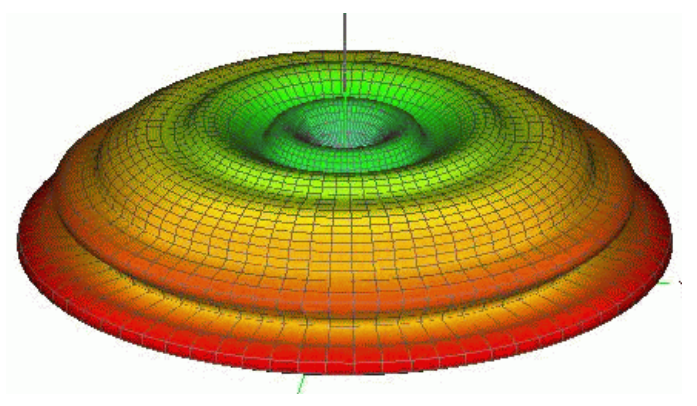

Fig. 4. The dipole antenna pattern with lunar ground effects.

\section{B. Lunar Ground Material}

Figs. 5 and 6 show the comparison of the computed signal strengths versus various ground materials at 401 $\mathrm{MHz}$. The half-wave dipole antenna and vertical polarization are assumed for transmitter and receiver. The transmit power is normalized to 1 Watt without circuit loss. Both of the transmitting and receiving antennas are placed at the same height of $6 \mathrm{~m}$ and $2 \mathrm{~m}$ above the ground. The free space case is without a ground effect. The PEC case is with a perfect electric conducting ground.

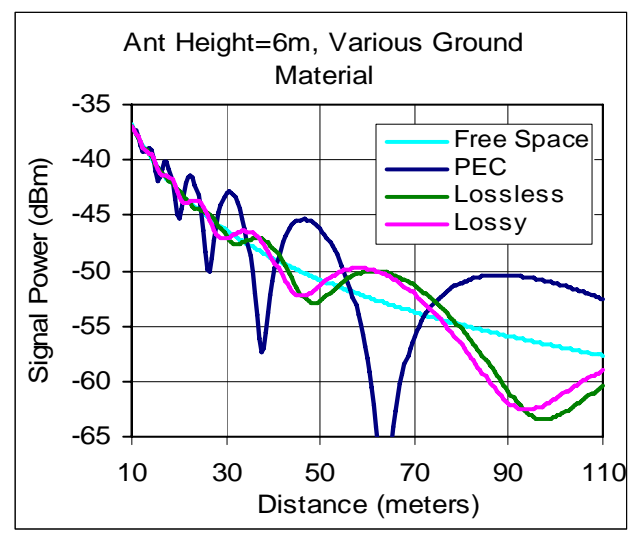

Fig. 5. The received UHF signal power oscillates with various lunar ground materials at an antenna height of $6 \mathrm{~m}$.

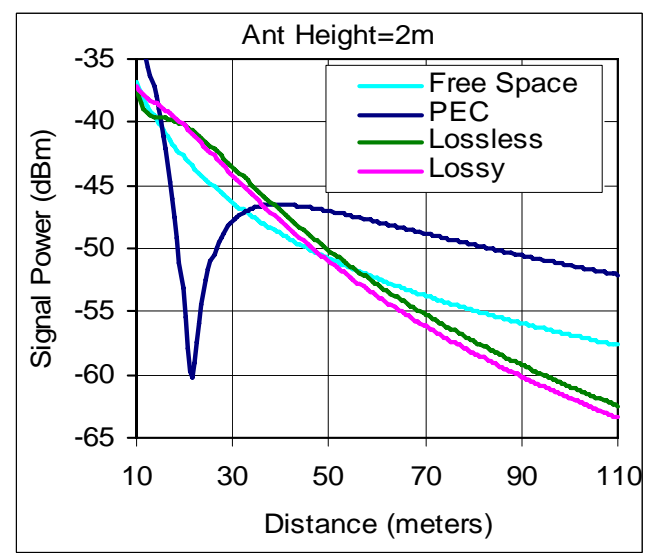

Fig. 6. The received UHF signal power oscillates with various lunar ground materials at an antenna height of $2 \mathrm{~m}$.

The ground reflections cause the signal oscillations. The strong reflections from the conducting ground give deep nulls. The signal signatures are similar and only slightly shift between low-loss (or lossless) and high-loss (or lossy) lunar ground. The signal strength is about $1.5 \mathrm{~dB}$ lower for the high-loss ground at a range distance of $100 \mathrm{~m}$. The loss will increase at long-range distance. In general, the averaging signal strengths at long range with lunar ground effects are lower than the free space, as shown in Fig. 6. The lunar ground acts as a partial reflector and partial absorber to the RF signals.

\section{Antenna Height}

Fig. 7 shows the comparison of the signal strengths for antenna at various heights above the lunar ground surface. The averaging signal strengths increase with increasing antenna heights. As the curves show, higher antennas lead to smaller path loss since less blockage and better ground clearance produce closer to a line-of-sight free space condition. Therefore, there are advantages to mounting the antenna at a higher position to reduce the path loss due to the lunar ground effects.

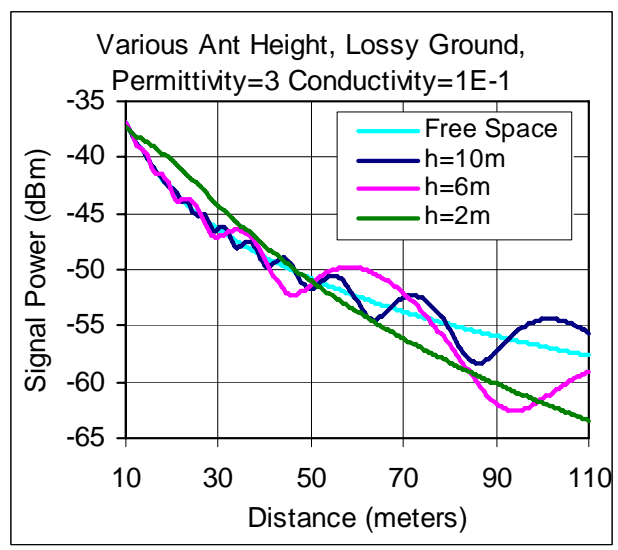

Fig. 7. The received UHF signal power oscillates due to lunar ground effects with various antenna heights. 


\section{Frequency Effects}

Fig. 8 shows the comparison of the computed UHF signal path loss with and without the lunar ground effects. The ground effects cause a path loss that is more severe than in the free space at a range greater than $50 \mathrm{~m}$. The transmitter and receiver antennas are vertical dipoles at $2 \mathrm{~m}$ above ground. The propagation loss is $10 \mathrm{~dB}$ more than free space at $200 \mathrm{~m}$. Fig. 9 shows the comparison of the computed Sband signal path loss with and without the lunar ground effects. The ground effects cause a path loss that is $5 \mathrm{~dB}$ less than in the free space at a range of $100 \mathrm{~m}$ due to the constructive interferometer effects from the ground reflection. However, the path loss can be worse than the free space at long-range distance, as shown in the following section.

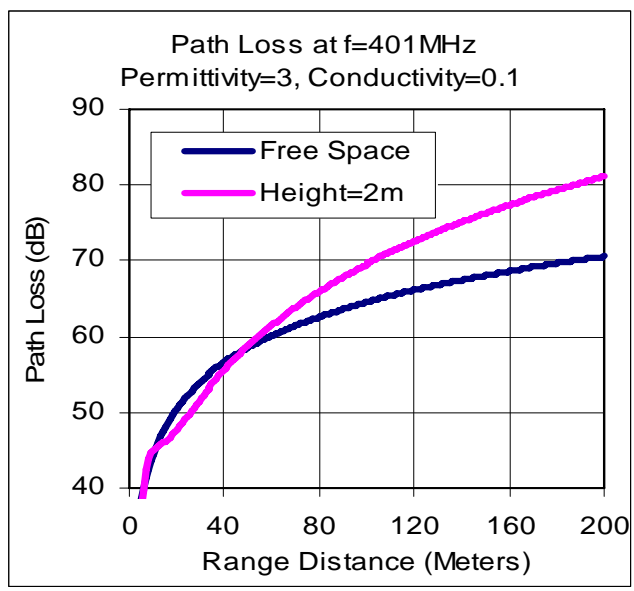

Fig. 8. The path loss of the UHF signal is greater than free space at range $>50 \mathrm{~m}$.

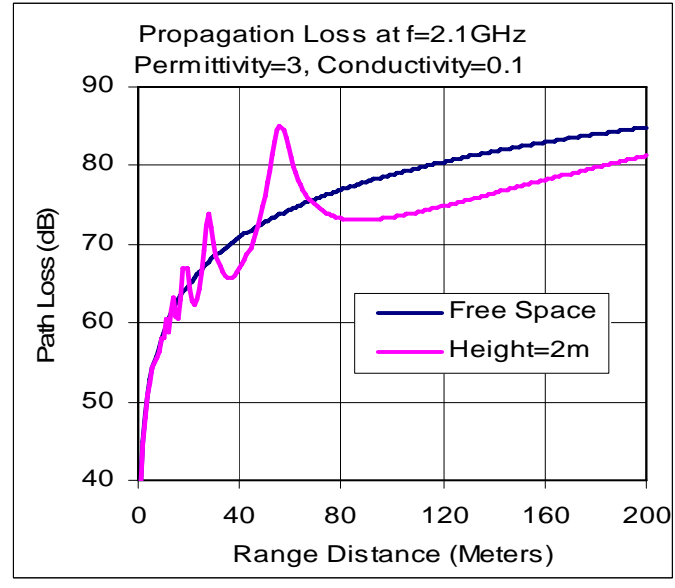

Fig. 9. The path loss of the S-band signal is less than free space due to the lunar ground effects at $80-200 \mathrm{~m}$.

Fig. 10 shows the comparison of the computed path loss between UHF and S-band signals. The path losses of UHF and S-band signals approach each other at distances greater than $180 \mathrm{~m}$. The path loss is independent of frequencies at long-range distances in lunar environment, as shown in Fig.
10. The path loss in the lunar environment is contradicted to the free space path loss. In free space, path loss is a strong function of frequency.

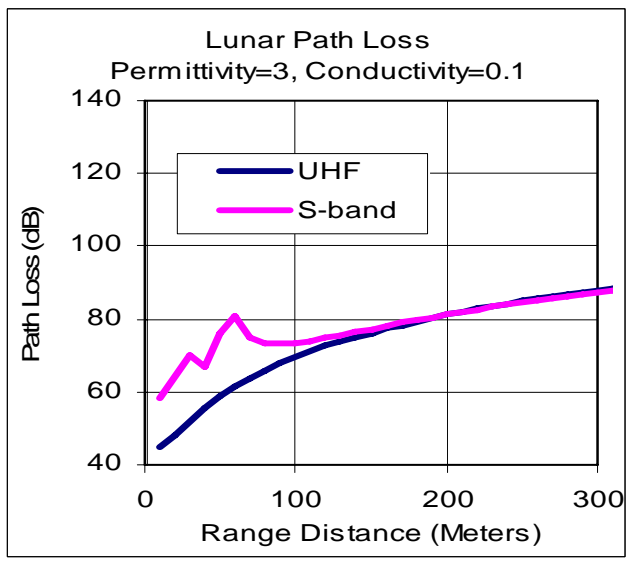

Fig. 10. The path losses of UHF and S-band signals approach each other at distances greater than $180 \mathrm{~m}$.

\section{E. Path Loss}

The computed results indicate that when the range distance is small, the received signal strengths oscillate with peaks and nulls due to the interferometer from the ground reflection. In the short-range region, path loss increases in proportion to $1 / \mathrm{R}^{2}$, as in free space. However, when the range distance is large, path loss increases in proportion to $1 / R^{4}$, as shown in Figs. 11 and 12 . This is because the reflection coefficiency is approaching -1 at an angle of near-grating incidence that causes cancelling between direct field and reflected field. The breakpoint is where the path loss transitions from square law to fourth law.

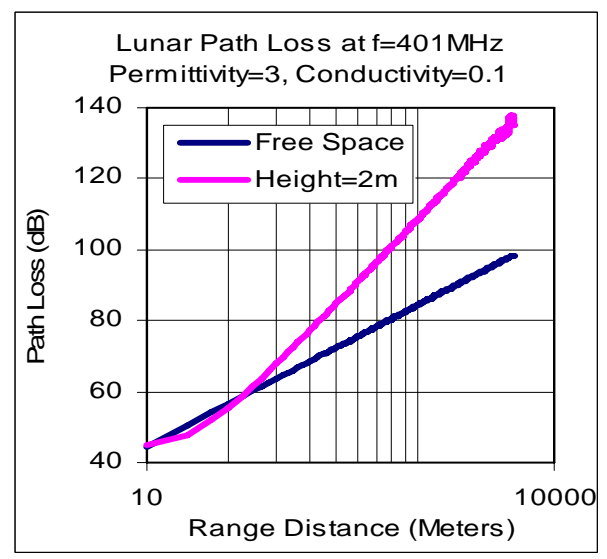

Fig. 11. The UHF signal path loss increases in proportion to $1 / \mathrm{R}^{2}$ in short range and to $1 / \mathrm{R}^{4}$ in long range. 


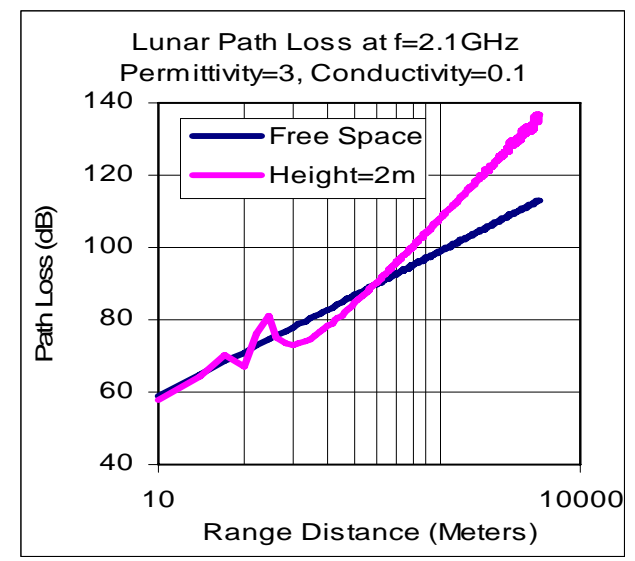

Fig. 12. The S-band signal path loss increases in proportion to $1 / \mathrm{R}^{2}$ in short range and to $1 / \mathrm{R}^{4}$ in long range.

The computed results indicate that the breakpoint is a function of frequency and antenna height, as shown in Figs. 13 and 14. For the UHF systems, with antenna height of 2 $\mathrm{m}$, the breakpoint is taking place at about $50 \mathrm{~m}$. For the Sband signals, it is at about $320 \mathrm{~m}$. The computed data indicate that the pass loss decreases with increasing antenna height, as shown in Figs. 13 and 14, at a long-range distance that is greater than $60 \mathrm{~m}$ for UHF and $300 \mathrm{~m}$ for Sband. The breakpoint, where the path loss turns to fourth power, is also moved farther with a higher antenna mounting location. However, deep nulls are observed in Fig. 14 for higher antenna height due to the signal cancelling from the ground reflections.

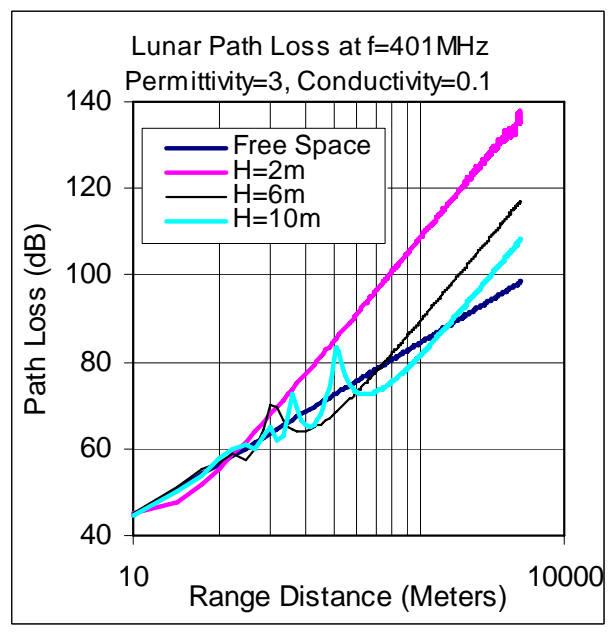

Fig. 13. The UHF signal path losses vs. various antenna heights.

The computed path loss data also show the independence of the frequency at long-range distances, as shown in Fig. 15. Note that the free space path loss is a strong function of frequency. Thus, in the circuit margin calculations for the lunar wireless systems, the commonly used free space loss formula cannot be used in the long-range communication link calculations due to the lunar ground effects. Otherwise, significant errors will occur in the computed link margin.

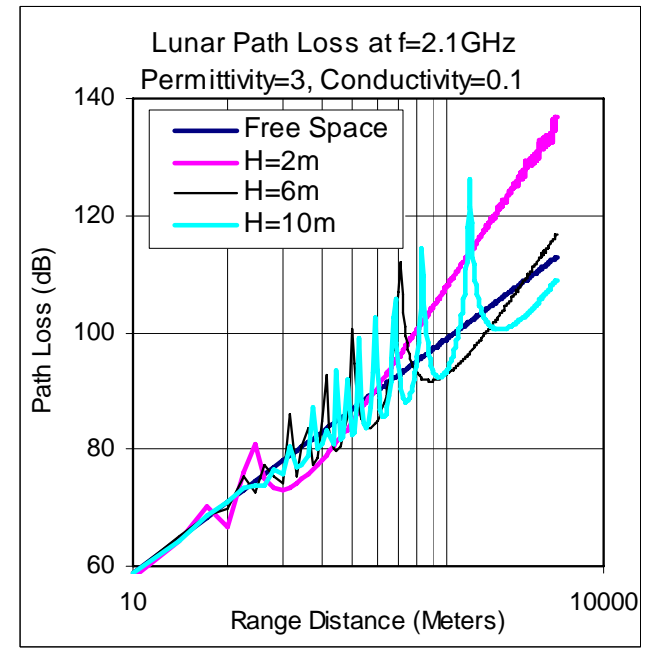

Fig. 14. The S-band signal path losses vs. various antenna heights.

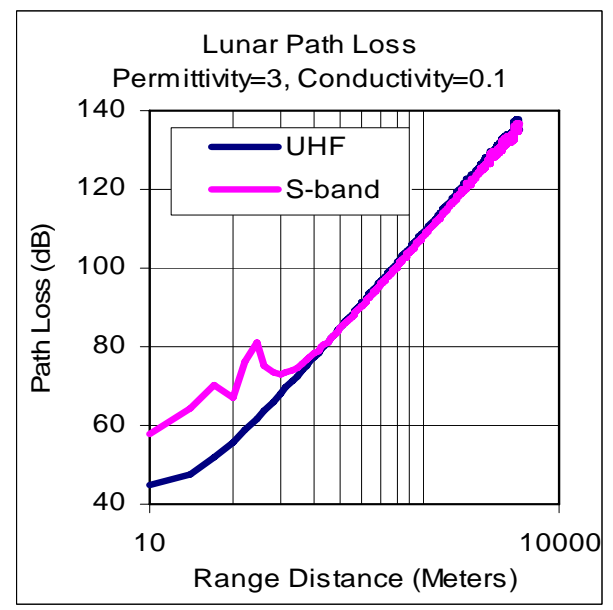

Fig. 15. The path loss is independent of frequency at long-range distances.

\section{3-D LUNAR CRATER TERRAIN}

The propagation in the Meteor Crater environment was investigated. Signal strengths were computed, including reflections and diffractions from the 3-D terrain model. The Meteor Crater in Arizona was used to simulate a lunar crater, as shown in Fig. 16. Terrain material was altered to resemble lunar soil. The GTD modeling technique is computationally efficient for electrical large vehicle and detail 3-D terrain models. Detail 3-D lunar terrain was modeled with complex dielectric constant for terrain material. Lunar vehicle and astronauts can be included in the model. Various types of antennas can be modeled. Multiple reflections and diffractions are included in the signal strength computations, as shown in Fig. 17. Signal strength can be mapped in a specified region, including the shadow region, for RF coverage analysis. 


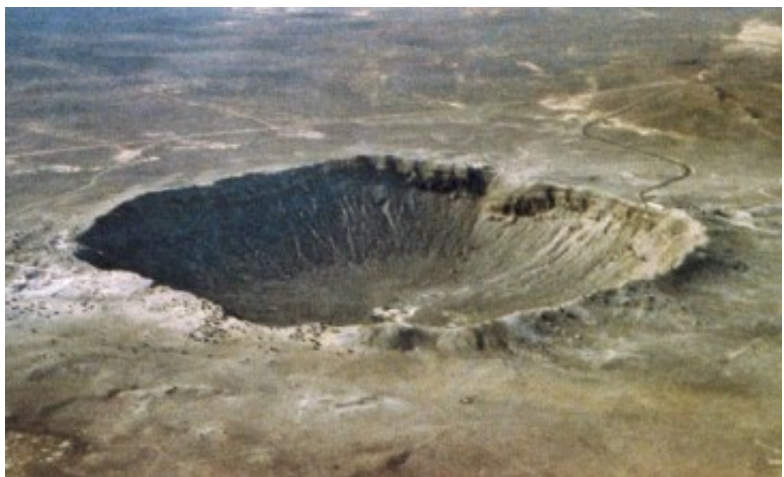

Fig. 16. The Meteor Crater modeled in the analysis.

\section{A. Signal Strengths}

As shown in Fig. 17, significant reflections exist in a crater environment. Fig. 18 shows sample results in the crater environment. The transmitter was placed at the edge of the crater ring. A receiver grid covered the bottom of the crater's surface. The receiver grids were placed in reference to terrain elevations. The shadowing and interferometer effects are observed in the simulation results. If the transmitting antenna is located away from the ring, the crater terrain can cause significant signal drop due to shadowing, as illustrated in the blue color region in Fig. 18.

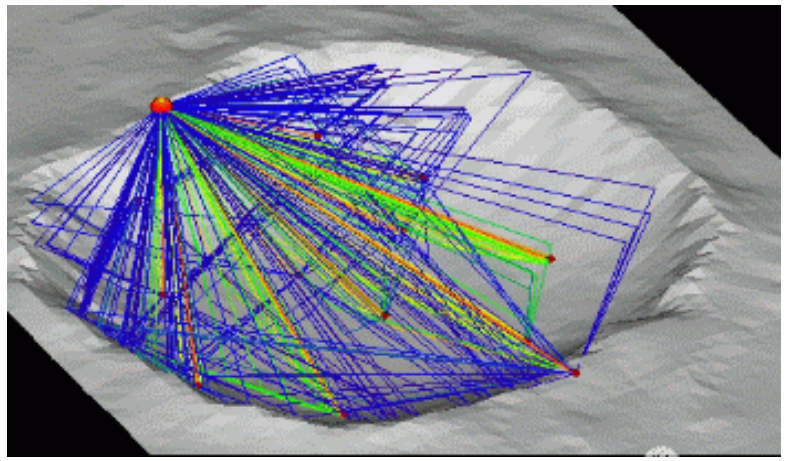

Fig. 17. Propagation paths for receiver points at various locations.

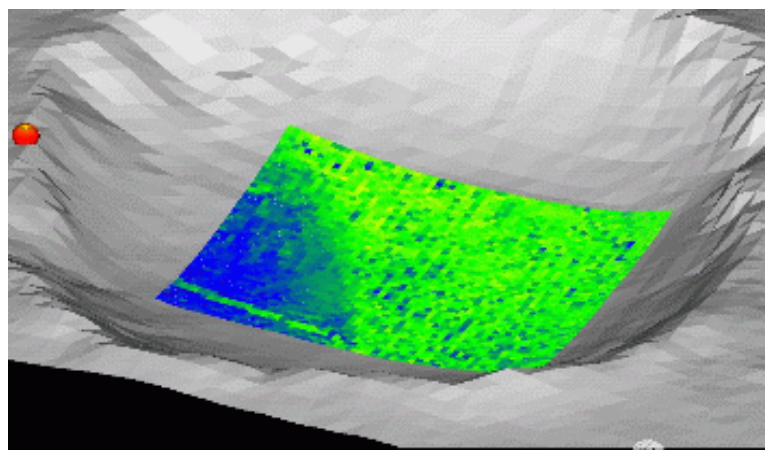

Fig. 18. Signal strength in horizontal receiver grid through crater.

\section{B. Signal Delay}

Signal delay is a major concern from the multipath for a high-data-rate wireless system. Due to the reflections off the lunar terrain, many indirect rays reach the receiver at longer travel time via longer indirect paths than the direct ray via shorter direct path, as shown in Fig. 19. The receiver may see a mix of delayed previous symbol and current symbol. This leads to intersymbol interference (ISI) at the receiver, which can cause bit errors.

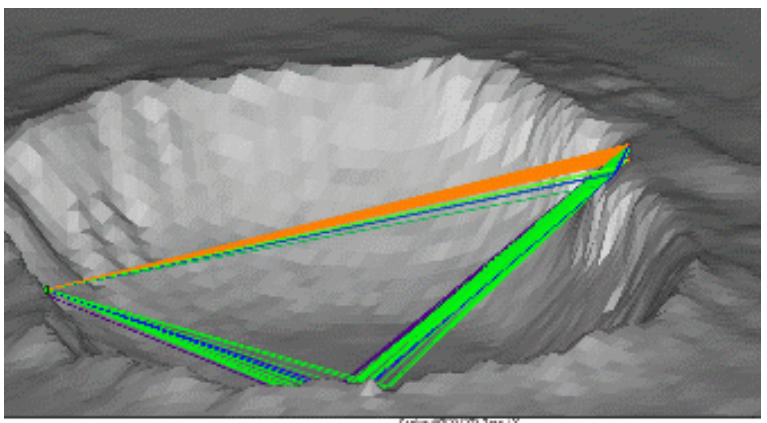

Fig. 19. The reflected signals can be delayed and cause ISI for high data rate systems.

For a reliable communication, the transmitted data rate should be much smaller than the inverse of the Root Mean Square delay spread. To decrease the probability of ISI, the symbol length cannot be shorter than the signal delay. A long symbol length will limit the maximum data rate that can be transmitted by the wireless systems. Fig. 20 shows the signal delays are about 160 nanoseconds (ns) for the selected case. The delay specification for many commercial 802.11-g products is $70 \mathrm{~ns}$ or less. This delay may put a limit on the maximum data rate that can be achieved in the lunar crater environment. Increasing antenna height will increase the signal delay. When the antenna is raised, it will see more reflecting objects at greater field of view areas with longer delay paths. On the other hand, a higher antenna reduces path loss due to the ground reflections and signal cancellations. It is a tradeoff between path loss and signal delay for a given antenna height and for a specific terrain environment in designing a high-data-rate wireless system.

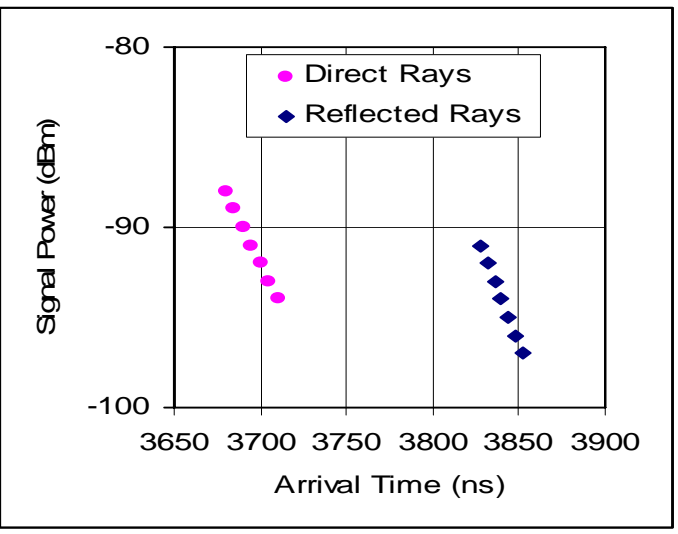

Fig. 20. The reflected signals are delayed by about 160 ns. 


\section{CONCLUSION}

Propagation characteristics and signal distribution are the essential parameters for wireless network planning and systems performance analysis. This paper presents the propagation analysis of the lunar wireless communication and sensor systems taking into account the 3-D terrain multipath effects. It is observed that the propagation characteristics are significantly affected by the presence of the lunar terrain.

The obtained results indicate that lunar surface material, terrain geometry, and antenna location are the important factors affecting the propagation characteristics of the lunar wireless systems. The antenna pattern is distorted due to lunar ground effects. Lunar ground also causes higher propagation loss and lower signal strength than in free space. Raising antenna height improves signal levels. Data show a higher path loss for higher frequency signals at short range. However, the path loss is frequency independent at long range due to the ground effects. The crater terrain is common on the lunar surface, which can cause significant signal drop due to shadowing. Signal delay could be a concern in a crater environment. The signal delay due to the lunar terrain can put a limit on the maximum data rate that can be achieved in the lunar environment.

The path loss in the lunar environment can be much more severe than the free space propagation and is greatly affected by the antenna height, surface material, and operating frequency. Test data from Earth terrain may not be applicable due to foliage/vegetation effects. The results from this paper are important for the lunar wireless system link margin analysis to determine the limits on the reliable communication range, achievable data rate, and $\mathrm{RF}$ coverage performance at planned lunar base worksites.

\section{REFERENCES}

[1] A. G. Longley and P. L. Rice, "Prediction of tropospheric radio transmission loss over irregular terrain," U.S. Department of Commerce, ESSA Rep. ERL-79-ITS-67, 1968.

[2] Y. Okumura, E. Ohmori, T. Kawano, and K. Fukuda, "Field strength and its variability in VHF and UHF land-mobile radio service," Rev. Elect. Commun. Lab., vol. 16, no. 9-10, Sept.-Oct. 1968.

[3] M. Hata, "Empirical formula for propagation loss in land mobile radio services,” IEEE Trans. Veh. Technol., vol. VT-29, no. 3, Aug. 1980

[4] M. Lebherz, W. Wiesbeck, and W. Krank, "A Versatile Wave Propagation Model For The VHF/UHF Range considering ThreeDimensional Terrain,” IEEE Transactions on Antennas and Propagation, vol. 40, pp. 1121-1131, Oct. 1992.

[5] V. Hrceg, et al., "An Empirically Based Path Loss Model for Wireless Channels in Suburban Environments,” IEEE Journal on Selected Areas in Comm., vol. 17, pp. 1205-1211, July 1999.

[6] H. Xia, H. Bertoni, L. Maciel, A. Lindsay-Stewart, and R. Rowe, "Radio propagation characteristics for line-of-sight microcellular and personal communications,” IEEE Trans. Antennas Propag., vol. 41, no. 10, Oct.1993.

[7] D. Erricolo and P. L. E. Uslenghi, "Propagation Path Loss - A Comparison between Ray-Tracing Approach and Empirical Models," IEEE Trans. Ant. And Propag., vol. 50, pp. 766-768, May 2002.
[8] G. Patwari, D. Durgin, T. S. Rappaport, and R. J. Boyle, "Peer to Peer Low Antenna Outdoor Radio Wave Propagation at $1.8 \mathrm{GHz}$," 49th IEEE Vehicular Technology Conference, Houston, TX, May 1999.

[9] R. Luebbers, V. Ungvichian, and L. Mitchell, "GTD terrain reflection model applied to ILS glide slope," IEEE Trans. Aerosp. Electron. Syst., p. 11, Jan. 1982.

[10] R. G. Kouyoumjian and P. H. Pathak, "A uniform geometrical theory of diffraction for an edge in a perfectly conducting surface," Proc. IEEE, vol. 62, no. 11, pp. 1448-1461, Nov. 1974

[11] R. J. Marhefka and J. W. Silvestro, "Near zone - basic scattering code user's manual with space station applications," NASA CR181944, Dec. 1989.

[12] S. Hwu, J. Dobbins, Y. Loh, C. Sham, and Q. Kroll, "Space Shuttle UHF Communications Performance Evaluation," IEEE Aerospace and Electronic Systems Magazine, pp. 9-14, vol.20, Oct. 2005. 